\title{
Laboratory-generated Coronal Mass Ejections
}

\author{
Christopher Watts ${ }^{1}$, Yue Zhang ${ }^{1}$, Alan Lynn, ${ }^{1}$ \\ Ward Manchester ${ }^{2}$ and C. Nick Arge ${ }^{3}$ \\ ${ }^{1}$ University of New Mexico, Albuquerque, NM 87131, USA; \\ ${ }^{2}$ University of Michigan, Ann Arbor, MI 48109, USA; \\ ${ }^{3}$ Air Force Research Laboratory, Kirtland AFB, Albuquerque, NM 87117, USA
}

\begin{abstract}
We have begun a series of laboratory experiments focused on understanding how coronal mass ejections (CME) interact and evolve in the solar wind. The experiments make use of the Helicon-Cathode (HelCat) plasma facility, and the Plasma Bubble eXperiment (PBeX). $\mathrm{PBeX}$ can generate CME-like structures (sphereomak geometry) that propagate into the highdensity, magnetized background plasma of the HelCat device. The goal of the current research is to compare CME evolution under conditions where there is sheared flow in the background plasma, versus without flow; observations suggest that CME evolution is strongly influenced by such sheared flow regions. Results of these studies will be used to validate numerical simulations of CME evolution, in particular the 3D BATS-R-US MHD code of the University of Michigan. Initial studies have characterized the plasma bubble as it evolves into the background field with and without plasma (no shear).
\end{abstract}

Keywords. Plasmas, Coronal Mass Ejections(CMEs), Laboratory

\section{Introduction}

Coronal mass ejections (CMEs) are the most stunning activity of the solar corona in which typically $10^{12}-10^{13} \mathrm{~kg}$ of plasma is hurled into interplanetary space with a kinetic energy of the order $10^{24}-10^{25} \mathrm{~J}$. They are of intense practical interest, as ultimately they are the cause of major geomagnetic storms here on Earth. Thus, it is important to understand the evolution of the CME as it propagates from the Sun to Earth through the solar wind. To this end, we have developed a laboratory experiment to "simulate" the dynamics of CME-like structures and their interactions with a magnetized background plasma.

There are three major goals to this project. First, we will attempt to gain a much better understanding of the dynamics of CMEs in the corona through direct and controlled laboratory experimentation that can, in many ways, realistically replicate the conditions found in the Sun's corona from 2-10 solar radii $\left(R_{\odot}\right)$. Second, we will use the University of Michigan state-of-art 3D MHD numerical model to evaluate and validate how well it can simulate the results generated by the plasma physics experiment. And then finally, determine what new physics, or improvement in physics, may need to be added to the numerical model in order to more realistically simulate actual CMEs. The overall goal is to further develop and validate a sophisticated numerical code that can be applied with more confidence to a broad spectrum of interacting plasmas situations, including especially CME-solar wind interactions. 

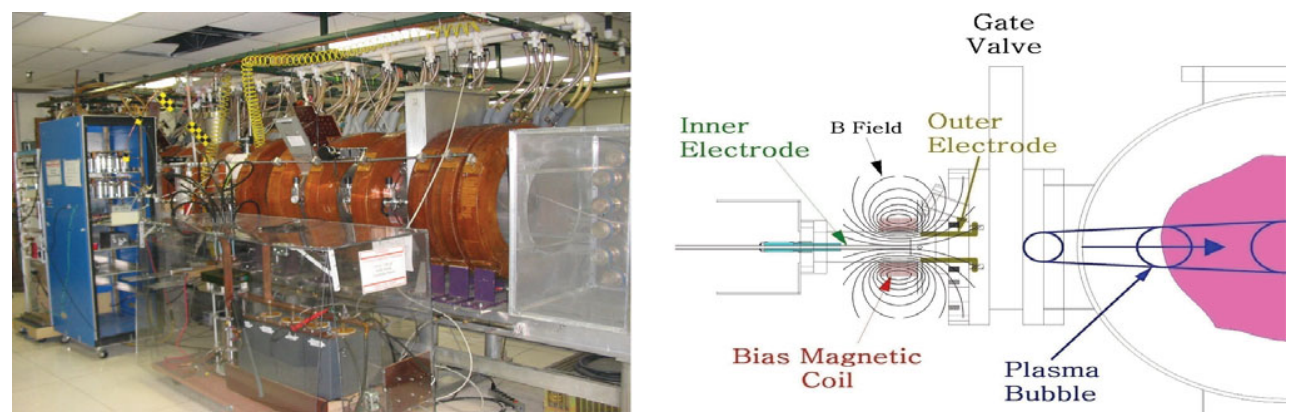

Figure 1. Left: Side view HelCat plasma device, with the PBeX electronics in the foreground. Right: Schematic of the PBeX gun system

\section{Experimental Apparatus}

The Plasma Bubble eXperiment (PBeX) magnetized coaxial gun is mounted on one side wall of the HelCat vacuum chamber (see Figure 1). HelCat is a large plasma facility (Christopher Watts 2005, Lynn et al., 2009), $4 \mathrm{~m} \mathrm{long,} 50 \mathrm{~cm}$ diameter with two plasma sources: an RF helicon and a thermionic cathode. The field strength and large diameter are sufficient to insure that the ions are magnetized. A large number of both large and small ports allows for easy diagnostic access. The two sources are able to generate background plasmas over an extensive regime of plasma operating parameters for a wide range of basic plasma studies, with relevance to fusion and astrophysical plasmas. These studies include turbulence dynamics, sheared flows, and Alfvénic waves.

Typically, the plasma is $\sim 100 \%$ ionized. However, the sources can be adjusted to create a large neutral fraction, if desired. HelCat also makes use of several sets of electrodes/grids that can be biased to induced sheared plasma flows. Of particular interest in our studies is the propagation of CME-like structures through regions of sheared flow.

Also in Figure 1 is a schematic depiction of the PBeX gun. It injects a high density, magnetized, supersonic plasma jet into the magnetized background plasma generated independently in the HelCat device. This can, in effect, simulate the evolution of a CME into the solar wind. The central component of PBeX is coaxial plasma gun designed to launch a magnetized plasma bubble or jet with various magnetic configurations. Of particular interest for this project is spheromak geometry. The jet has an initial density of $\sim 10^{20} / \mathrm{m}^{3}$, a factor of $10-10^{4}$ greater than the background plasma density; the magnetic field can be adjusted to up to 2 orders of magnitude larger than that of the background plasma. Thus, the bubble expands into the background at supersonic and super-alfvénic speeds, generating a shock front at the interface. Depending on the orientation of the background magnetic field, various Alfvénic (magnetic) shock configurations can be obtained, along with a range of magnetic reconnection geometries.

The laboratory is equipped with a wide array of diagnostic capabilities to allow us to study the PBeX dynamics. These include a fast imaging camera (1.5 ns gate time) for global visualization, a $4 \mathrm{~m}$ spectrometer for detailed ion temperature measurements, 3D magnetic and electrostatic probe arrays for localized magnetic field, electron temperature and ion density characterization, two microwave interferometers for chord averaged density measurements, and a microwave reflectometer for localized density measurements. Thus we can characterize the expanding plasma bubble "CME" with unprecedented detail that would only be achieved with real CMEs in space using a large constellation of spacecraft. 

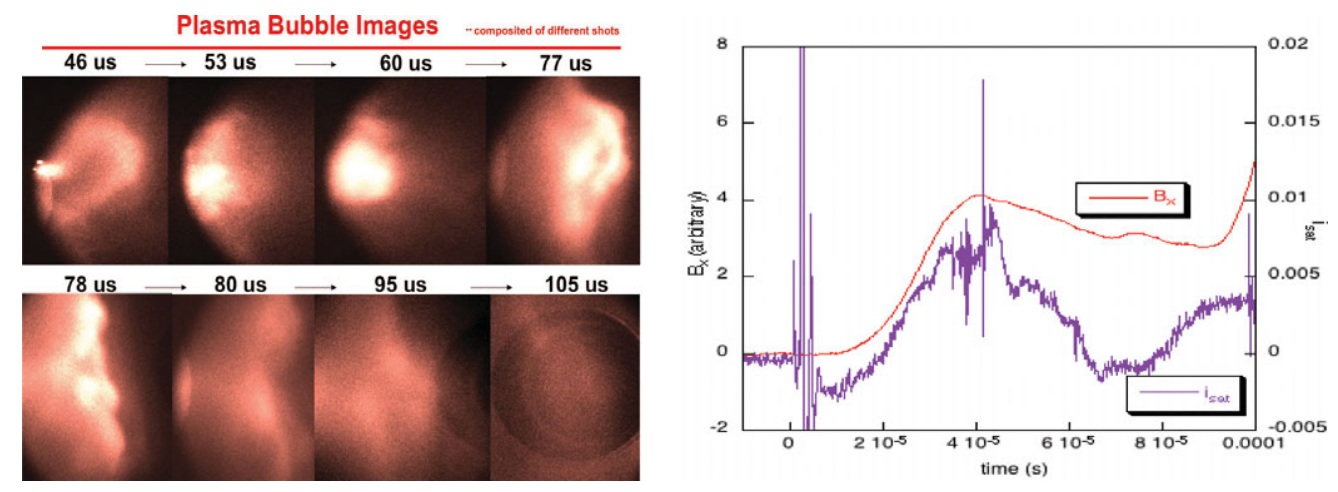

Figure 2. Left: Evolution of the PBeX plasma bubble in HelCat. Though the image sequence is from several different shots, the dynamics are very reproducible. Right: Plot of magnetic field and density (Isat) evolution during bubble expansion. At startup the two traces overlay, indicating the frozen flux in the expanding bubble shell

Solar prominence and flare formation have been modeled in the laboratory by several groups (Bellan, 1997, Hsu et al., 2005) . These have used plasma generation mechanisms designed to create twisted flux ropes, and are focused on understanding formation and flux rope dynamics early in time. In contrast to this work, we are not interested in the formation dynamics of the CME. Rather, we specifically focus on the CME evolution long after the initial formation, as it is propagating and interacting with the solar wind. Here, the background plasma - not present in their experiments - plays a crucial role.

$\mathrm{PBeX}$ has been in operation only one year, and initial studies of the expanding plasma bubble have demonstrated the essential dynamics. Density, temperature and magnetic field measurements indicate the bubble has the expected parameters. Figure 2 shows a series of photographs taken with the fast imaging camera, depicting the evolution of the plasma bubble as it expands in the background HelCat plasma. Qualitatively, at least, the structure is similar to that of a CME as it expands into the interplanetary medium.

\section{Modeling Connection}

Our modeling effort will make use of the University of Michigan state-of-the-art 3-D MHD BATS-R-US code (Powell et al., 1999, Groth et al., 2000) to couple our PBeX laboratory experiments to solar wind observations. In the typical region of interest for CME study, 2-10 $\left(R_{\odot}\right)$, coronal particle densities range from $5 \times 10^{11}$ to $10^{13}$ particles per cubic meter. Beyond $3\left(R_{\odot}\right)$ the plasma is essentially collisionless such that the ionization states remain frozen in as wind expands and cools. By contrast, the plasma experiments are performed with particle densities orders of magnitude higher, where particle interactions are dominated by collisions. The question naturally arises: Can a meaningful comparison be made between the experiments, coronal mass ejections and ideal MHD simulations?

Direct observations clearly indicate that the low-density plasma of the solar wind behaves in many ways like a much denser collision-dominated plasma. This fact is most obvious at the planetary bow shocks that form in the solar wind. The particle coupling and dissipation necessary for shock formation is attributed to wave-particle interaction, which makes the plasma behave like a much denser plasma in which coupling occurs directly by particle collisions. Shock-capturing MHD simulations performed with BATSR-US accurately reproduce the behavior of CME-driven shocks in the solar wind. An example is found in Manchester et al. (Manchester et al., 2008), where they accurately 
simulate the structure of a CME-driven shock observed with the LASCO C3 coronagraph onboard the SOHO observatory.

Conversely, the PBeX bubble evinces behavior of a much less collisional Ideal MHD plasma. Figure 2 also compares the evolution of the magnetic field and density (ion saturation current) measured at a single point as the bubble evolves past. Such measurements of density and magnetic field indicate that, at least initially (between 0 and $40 \mathrm{~s}$ ), the field and plasma at the bubble's leading edge flow together, i.e., the flux is "frozen" into the expanding plasma. This, in spite of what the (low) magnetic Reynold's number might indicate. The magnetic Reynold's number $R_{s}$, and Lundquist number $\mathrm{S}$, both characterize the coupling of the plasma and magnetic flux; a large value indicates the magnetic field is "frozen in" to the plasma. However, the highly collisional nature of the high-density laboratory plasma coupled with the short scale lengths artificially deflate Rm, which in HelCat/PBeX is about $100-1000$ as opposed to $>10^{6}$ in the solar wind, despite the fact that experiments confirm the flux is indeed convected with the plasma (or vice versa).

\section{Perspective}

Initial results of the PBeX experiment on the HelCat device, aimed at modeling CME propagation through the solar wind, have been presented. These measurements indicate that $\mathrm{PBeX}$ is highly relevant to understanding the less collisional, larger scale CME event, where Ideal (collisionless) MHD describes the plasma dynamics, and is also relevant for benchmarking the BATS-R-US code. Near term work will focus on the interaction of the bubble with the background plasma in terms of the evolution of magnetic helicity, magnetic and thermal energy, and azimuthal rotation. Also these experiments will investigate the dependence of flux amplification on gun parameters as well as background plasma parameters such as the background thermal and magnetic pressure. Longer term, we plan investigations with two major thrusts: 1) Understanding magnetized shock formation and evolution at the boundary between the bubble and background plasma, and 2) Characterizing the bubble evolution in the presence of sheared flows in the background plasma.

\section{References}

Bellan, P. M., 1997, Proceedings of the 4th IPELS Workshop, Maui, Hawaii

Groth, C. P. T., De Zeeuw, D. L., Gombosi, T. I., \& Powell, K. G., 2000, Journal of Geophysical Research, 105, 25053

Hsu, S. C. \& Bellan, P. M., 2005, Phys. Plasmas, 12, 1

Lynn, A., Gilmore, M., Watts, C., Herrea, J., Kelly, R., Will, S., Shuangwei, X., Lincan, Y., \& Yue, Z., 2009, Rev. Sci. Inst., 80, 103501

Manchester IV, W. B., Vourlidas, A., Toth, G., Lugaz, N., Roussev, I. I., Sokolov, T. V., Gombosi, T. I., De Zeeuw, D. L., \& Opher, M., 2008, Astrophysical Journal, 684, 1448

Powell, K. G., Roe, P. L., Linde, T. J., Gombosi, T. I., \& De Zeeuw, D. L., 1999, Journal of Computational Physics, 154, 284

Watts, C., 2005, Rev. Sci. Inst., 75, 1975 